\title{
Procesos FIGARCH: Estimación de la volatilidad del tipo de cambio nominal del Perú
}

\author{
José Luis Briones Zúñiga ${ }^{1}$ Antonio Bravo Quiroz $z^{2}$
}

Resumen: En esta investigación se presenta una revisión teórica de la estructura y aplicación de modelos de naturaleza de memoria larga que combinan características de los procesos fraccionalmente integrados con los clásicos modelos GARCH obteniéndose de esta manera los modelos Autorregresivos con Heterocedasticidad Condicionada Fraccionalmente Integrado (FIGARCH) los cuales a través de la función impulso respuesta acumulativa permitió cuantificar el grado de persistencia del impacto de la innovación sobre la función de la varianza condicionada, es decir el elemento de persistencia en una serie caótica muy sensible a cambios en la condiciones iniciales asociadas al movimiento browniano fraccional. Para dicha aplicación se utilizó la variable tipo de cambio y mediante modelos de series de tiempo de memoria larga poder analizar la persistencia del efecto existente en la volatilidad de dicha serie.

Palabras clave: FIGARCH; Movimiento Browniano fraccional; persistencia.

\section{FIGARCH Processes: Estimation on volatility in exchange rates of Perú}

Abstract: This research presents a theoretical review of the structure and application of long memory nature models that combine characteristics of the fractionally integrated processes with the classic GARCH models, thus obtaining the autoregressive models with fractionally integrated conditioned heterocedasticity (FIGARCH) which Through the cumulative response impulse function, I can quantify the degree of persistence of the impact of innovation on the function of conditioned variance, that is, the element of persistence in a chaotic series very sensitive to changes in initial conditions associated with fractional Brownian motion. . For this application, the exchange rate variable was used, and by means of long memory time series models, the persistence of the existing effect on the volatility of said series could be analyzed.

Keywords: FIGARCH; fractional Brownian Movement; persistence.

Recibido: 04/05/2019. Aceptado: 20/05/2019. Publicado online: 26/12/2019.

CLos autores. Este artículo es publicado por la Revista PESQUIMAT de la Facultad de Ciencias Matemáticas, Universidad Nacional Mayor de San Marcos. Este es un artículo de acceso abierto, distribuido bajo los términos de la licencia Creative Commons Atribucion-No Comercia-Compartir Igual 4.0 Internacional.(http://creativecommons.org/licenses/by-nc-sa/4.0/) que permite el uso no comercial, distribución y reproducción en cualquier medio, siempre que la obra original sea debidamente citada. Para información, por favor póngase en contacto con revistapesquimat.matematica@unmsm.edu.pe

\footnotetext{
${ }^{1}$ UNMSM, Facultad de Ciencias Matemáticas e-mail: likaseconometria@gmail.com

${ }^{2}$ UNMSM, Facultad de Ciencias Matemáticas. e-mail: abravoq@unmsm.edu.pe
} 


\section{Introducción}

La modelización del proceso de volatilidad del tipo del cambio es una pieza que da solidez a la estructura de política económica de una nación generando bases para una dinámica democrática necesarias para el desarrollo de un sistema pero debido a la naturaleza heterocedástica de los retornos de la tasa de cambio de corto plazo, los clásicos modelos que suponen estacionariedad y homocedasticidad no son los más idóneos para modelarla. Siendo dicha modelización de gran importancia en la toma de decisiones macroeconómicas y en las últimas décadas motivo de investigaciones en el mercado financiero.

En investigaciones como el de Baillie y Bollerslev (1989,1991), Hsieh(1988), Engle(1990), Vlaar y Palm(1993), entre otros, han utilizado la metodología ARCH y GARCH para la modelización del proceso de volatilidad con la estructura econométrica del modelo gaussiano $\operatorname{GARCH}(1,1)$ suponiendo a la innovación unitaria de la varianza condicionada como una distribución gaussiana mesocúrtica y simétrica, por tal motivo dicho modelo es el más empleado para estudiar la volatilidad, sin embargo un número importante de estudios, empleando base de datos diarias han encontrado evidencia sobre la persistencia en la volatilidad es decir dicha innovación es más parecida a una distribución T o una Levy Pareto con colas más pesadas en relación a una distribución normal aproximando el proceso por medio de modelos IGARCH, FIGARCH e HYGARCH.

Por lo tanto proponiendo una evolución en la estructura de teoría basada en un ruido gaussiano siendo una distribución estable y ergódica con sus pilares el teorema del límite central y la ley de los grandes números, en la cual si existe un impacto este se disemina, es decir es absorbido por la sumatoria de variables aleatorias e independientemente distribuidas o por la posible existencia de cierta correlación espuria ".... en algunos casos, el cuadro general de independencia fue presentado pero en otros habian signos de dependencia como se reflejan en las correlaciones seriales. Sin embargo,tales correlaciones seriales presentes en las series son muy débiles como para disponer de cualquier posibilidad de poder utilizarlas para prediccion".(Kendall,1953); a una distribución más estable que la gaussiana pero no ergódica debido a su jerarquización propuesta por el matemático polaco de origen judio Benoit Mandelbrot(1963), en la cual dicho impacto

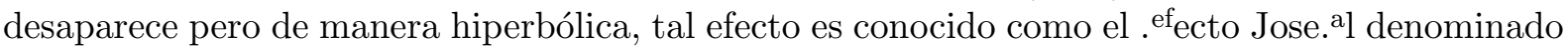
ruido gaussiano fraccionario.

Por lo tanto el propósito es la de desarrollar un modelo mucho más flexible para capturar la dependencia temporal observada de la volatilidad en la dinámica de un fenómeno. De la idea anterior surgió el modelo FIGARCH, propuesto inicialmente por Bollerslev, Baillie y Mikkelsenen 1996, Granger y Ding en 1996 y Chung en 1999, se basa en el hecho de pensar que los retornos cuadráticos poseen memoria larga y por lo tanto que estos impactan en la varianza condicional, siendo esta persistente y mucho mayor que el modelo ARFIMA, por lo que el impacto de un shock desaparece muy lentamente manifestándose en la lenta convergencia de la estabilización de la varianza condicionada.

Por lo tanto esta investigación tiene por objetivo dar a conocer las principales caracteristicas del modelo FIGARCH asi como su aplicación.

\section{Estructura del proceso FIGARCH}

Según Fan y Yao (2005), la ACF de un proceso ARMA estacionario satisface la desigualdad:

$$
|\rho(k)| \leqslant C r^{k} k=1,2,3, \cdots
$$


donde $C>0$ y $r \in[0,1]$, son constantes, es decir,

$$
\sum_{k=0}^{\infty}|\rho(k)|<\infty
$$

Por lo tanto, decimos un proceso absolutamente sumable se la denomina como un proceso de memoria corta. En cambio en los procesos de memoria larga la ACF decae muy lentamente, es decir hiperbólicamente, por tal motivo la formalidad matemática esta dado:

$$
\rho(k) \sim C k^{2 d-1} \text { cuando } k \rightarrow \infty
$$

donde $C \neq 0$ y $d<0,5$, de esta manera el efecto del shock disminuye muy lentamente a razón de $k^{2 d-1}$ entonces:

$$
\sum_{k=-\infty}^{\infty}|\rho(k)|=\infty
$$

la AFC no es absolutamente sumable. Si sabemos que el proceso $\varepsilon_{t}$, denota un proceso estocástico y "t", pertenece a un conjunto de sub índices por otro lado consideramos a $\Psi_{t}$, como el conjunto de información en el tiempo "t". Si consideramos: $\Psi_{t}=\sigma\left\{\ldots . ., \varepsilon_{t-2}, \varepsilon_{t-1}, \varepsilon_{t}\right\}$, es un sigma de álgebra sub conjunto del espacio muestral no vacío, entonces el conjunto formado por todos los subconjuntos del espacio muestral tambien es un sigma de álgebra.

Entonces se dice ser un proceso $\operatorname{ARCH}(q)$ :

$$
h_{t}=\alpha_{0}+\sum_{i=1}^{q} \alpha_{i} \varepsilon_{i-1}^{2}
$$

donde $\alpha_{i} \geqslant 0, i=1,2, \cdots, q$.

Si consideramos el siguiente enfoque

$$
\begin{gathered}
E\left(\varepsilon_{t} \mid \Psi_{t-1}\right)=0 \\
\operatorname{var}\left(\varepsilon_{t} \mid \Psi_{t-1}\right)=h_{t} \\
\varepsilon_{t} \mid \Psi_{t-1} \backsim N\left(0, h_{t}\right)
\end{gathered}
$$

Por lo tanto la varianza condicional se puede expresar :

$$
h_{t}=\sigma\left\{\varepsilon_{t-1}, \varepsilon_{t-2}, \varepsilon_{t-3}, \ldots, \varepsilon_{t-q}, \alpha\right\}
$$

donde $\mathrm{h}(\cdot)$ es una función no negativa y $\alpha_{i}=\sigma\left\{\alpha_{1}, \alpha_{2}, \alpha_{3}, \ldots, \alpha_{q}, \alpha\right\}$ ' es el vector de parámetros a estimar para modelar la varianza condicionada, entonces se podría representar al proceso estocástico de la siguiente manera:

$$
\varepsilon_{t}=h_{t}^{1 / 2} z_{t}
$$

A partir de $\varepsilon_{t}^{2}=h_{t} z_{t}^{2}$, si despejamos se tiene, $z_{t}^{2}=\frac{\varepsilon_{t}^{2}}{h_{t}}$, aplicando esperanza resulta

$$
E\left[z_{t}^{2} \mid \Psi_{t-1}\right]=E\left[\frac{\varepsilon_{t}^{2}}{h_{t}} \mid \Psi_{t-1}\right]
$$


De (4) se concluye, $E\left[z_{t}^{2} \mid \Psi_{t-1}\right]=1$, entonces, $\operatorname{var}\left(z_{t} \mid \Psi_{t-1}\right)=1$.

De (4) se tiene, $E\left[\varepsilon_{t} \mid \Psi_{t-1}\right]=E\left[z_{t} \mid \Psi_{t-1}\right] E\left[h_{t}^{1 / 2} \mid \Psi_{t-1}\right]$, se tiene, $0=E\left[z_{t} \mid \Psi_{t-1}\right] E\left[h_{t}^{1 / 2} \mid \Psi_{t-1}\right]$, se concluye $E\left[z_{t} \mid \Psi_{t-1}\right]=0$. Entonces:

$$
z_{t} \mid \Psi_{t-1} \backsim N(0,1)
$$

$\operatorname{con} E\left(z_{i}, z_{j}\right)=0, \forall i \neq j$

La innovación $z_{t}$ es independiente e idénticamente distribuidas es decir tiene una distribución gaussiana e incorrelacionadas serialmente con media 0 y varianza 1 por lo tanto es un movimiento browniano estándar(caso especial de proceso estocástico gaussiano), se puede concluir que $\varepsilon_{t}$ tambien es incorrelacionado serialmente, $\varepsilon_{t} \mid \Psi_{t-1} \sim N\left(0, h_{t}\right)$.

$$
\begin{gathered}
\varepsilon_{t}^{2}=h_{t} z_{t}^{2} \\
E\left[\varepsilon_{t}^{2} \mid \Psi_{t-1}\right]=E\left[z_{t}^{2} \mid \Psi_{t-1}\right] E\left[h_{t} \mid \Psi_{t-1}\right] \\
E\left[\varepsilon_{t}^{2} \mid \Psi_{t-1}\right]=h_{t}
\end{gathered}
$$

Entonces

$$
\varepsilon_{t} \mid \Psi_{t-1} \backsim N\left(0, h_{t}\right)
$$

$\operatorname{con} E\left(\varepsilon_{i}, \varepsilon_{j}\right)=0, \forall i \neq j$.

Si consideramos una extensión del proceso $\operatorname{GARCH}(\mathrm{p}, \mathrm{q})$, introducido por Bollerslev en el año de 1986 y Taylor en el año de 1986, obtenemos

$$
h_{t}=\alpha_{0}+\sum_{i=1}^{q} \alpha_{i} \varepsilon_{t-i}^{2}+\sum_{j=1}^{p} \gamma_{j} h_{t-j}
$$

donde: $p>0, q>0, \alpha_{0}>0, \gamma_{j} \geqslant 0, \alpha_{i} \geqslant 0, i=1,2,3, \cdots, q, j=1,2,3, \cdots, p$. además $\alpha(L)=\alpha_{1} L^{1}+\alpha_{2} L^{2}+\alpha_{3} L^{3}+\cdots+\alpha_{q} L^{q}, \beta(L)=\beta_{1} L^{1}+\beta_{2} L^{2}+\beta_{3} L^{3}+\cdots+\beta_{p} L^{p}$ son operadores de rezago

La función dada en (7) se puede expresarse del siguiente modo :

$$
h_{t}=\alpha_{0}+\alpha(L) \varepsilon_{t}^{2}+\beta(L) h_{t}
$$

con $\alpha_{0}>0, \alpha_{i} \geqslant 0, \gamma_{i} \geqslant 0, i=1,2, \cdots, q, j=1,2, \cdots, p$

Es importante observar que si consideramos :

1. $p=0$, tenemos un proceso $\operatorname{ARCH}(\mathrm{q}), h_{t}=\alpha_{0}+\sum_{i=1}^{q} \alpha_{i} \varepsilon_{t-i}^{2}$

2. $p=q=0$, tenemos un proceso ruido blanco.

$$
\varepsilon_{t} \mid \Psi_{t-1} \backsim N\left(0, h_{t}\right)
$$

Proceso $\operatorname{GARCH}(\mathrm{p}, \mathrm{q})$ estacionario a partir de la ecuación (5) reemplazamos en la esperanza tenemos:

$$
\operatorname{Var}\left(\varepsilon_{t}\right)=E\left(\varepsilon_{t}^{2}\right)=E\left(E\left(\varepsilon_{t}^{2} \mid \Psi_{t-1}\right)\right)=E\left(h_{t}\right)=\sigma_{t}^{2}
$$




$$
\operatorname{Var}\left(\varepsilon_{t}\right)=\alpha_{0}+\alpha_{1} E\left(\varepsilon_{t-1}^{2}\right)+\alpha_{2} E\left(\varepsilon_{t-2}^{2}\right)+\ldots+\alpha_{q} E\left(\varepsilon_{t-q}^{2}\right)+\gamma_{1} E\left(h_{t-1}\right)+\ldots+\gamma_{p} E\left(h_{t-p}\right)
$$

Si consideramos al proceso $\varepsilon_{t}$, estacionario, considerando la homocedasticidad en la serie

$$
E\left(\varepsilon_{t}^{2}\right)=E\left(\varepsilon_{t-k}^{2}\right), \forall k .
$$

Entonces

$$
\operatorname{Var}\left(\varepsilon_{t}\right)=\alpha_{0}+\alpha_{1} \operatorname{Var}\left(\varepsilon_{t}\right)+\alpha_{2} \operatorname{Var}\left(\varepsilon_{t}\right)+\cdots+\alpha_{q} \operatorname{Var}\left(\varepsilon_{t}\right)+\gamma_{1} \operatorname{Var}\left(\varepsilon_{t}\right)+\gamma_{2} \operatorname{Var}\left(\varepsilon_{t}\right)+\cdots+\gamma_{p} \operatorname{Var}\left(\varepsilon_{t}\right)
$$

y

$$
E\left(\varepsilon_{t}^{2}\right)=\frac{\alpha_{0}}{1-\left[\sum_{i=1}^{q} \alpha_{i}+\sum_{j=1}^{p} \gamma_{j}\right]}=\sigma_{t}^{2} .
$$

Por lo tanto consideramos la siguiente restricción:

$$
\sum_{i=1}^{q} \alpha_{i}+\sum_{j=1}^{p} \gamma_{j}<1
$$

Es importante mencionar que una señal de memoria larga en la serie se da cuando $\gamma_{j}$ se aproxima a 1, claro esta se debe realizar las pruebas tanto estadísticas paramétricas semiparametricas y no paramétricas para demostrar existencia de suficiente evidencia de persistencia en la serie.

Si incluimos la innovación unitaria $v_{t}$ como la innovación de la varianza condicional las cuales son variables aleatorias independientes e idénticamente distribuidas estacionarias e incorrelacionadas serialmente (shock):

$$
v_{t}=\varepsilon_{t}^{2}-h_{t}
$$

Consideramos la ecuación (5), entonces, $\varepsilon_{t}^{2}=h_{t} z_{t}^{2}$, desarrollando: $v_{t}=h_{t} z_{t}^{2}-h_{t}=h_{t}\left(z_{t}^{2}-1\right)$, recordando que $E\left(z_{i}, z_{j}\right)=0, \forall i \neq j$ y tambien $z_{t} \mid \Psi_{t-1} \backsim N(0,1)$. Entonces

$$
h_{t}=\alpha_{0}+\sum_{i=1}^{q} \alpha_{i} \varepsilon_{i-1}^{2}+\sum_{j=1}^{p} \gamma_{i} h_{t-j}
$$

Reemplazando la ecuación (10) en (11), resulta $h_{t}=\varepsilon_{t}^{2}-v_{t} \mathrm{y}$

$$
\varepsilon_{t}^{2}=\alpha_{0}+\sum_{i=1}^{q} \alpha_{i} \varepsilon_{t-q}^{2}+\sum_{j=1}^{p} \gamma_{j} \varepsilon_{t-j}^{2}-\sum_{j=1}^{p} \gamma_{j} v_{t-j}+v_{t}
$$

En el año 1986 Bollerslev y Engle, consideraron una clase de modelos GARCH(p,q) integrado denominado $\operatorname{IGARCH}(\mathrm{p}, \mathrm{q})$, en donde la dependencia a largo plazo de los incrementos del proceso es permanente a diferencia de los modelos $\mathrm{ARCH}(\mathrm{q})$ y $\mathrm{GARCH}(\mathrm{p}, \mathrm{q})$ en las cuales dichas persistencias desaparecen exponencialmente hacia cero.

En dicho modelo $\operatorname{IGARCH}(\mathrm{p}, \mathrm{q})$ se cumple:

$$
\sum_{i=1}^{q} \alpha_{i}+\sum_{j=1}^{p} \gamma_{j}=1
$$

A diferencia del modelo $\operatorname{GARCH}(\mathrm{p}, \mathrm{q})$

$$
\sum_{i=1}^{q} \alpha_{i}+\sum_{j=1}^{p} \gamma_{j}<1
$$


En los modelos IGARCH(p,q), el impacto del cuadrado de los shocks :

$$
v_{t-i}=\varepsilon_{t-i}^{2}-h_{t-i}
$$

para $i>0$ sobre el cuadrado de los residuos es persistente.

Por lo tanto en un modelo IGARCH(1,1), el modelo puede escribirse de la siguiente manera:

$$
\varepsilon_{t}=h_{t}^{1 / 2} z_{t}
$$

y

$$
h_{t}=\alpha_{0}+\beta_{1} h_{t-1}+\left(1-\beta_{1}\right) \varepsilon_{t-1}^{2}
$$

siendo $\beta \in\langle 0,1\rangle$. Dicho modelo $\operatorname{IGARCH}(1,1)$ es estacionario y ergódico.

Entonces el modelo IGARCH(p,q) implica una persistencia infinita de la varianza condicional para los shocks en el cuadrado de los retornos, una persistencia infinita de los shocks de volatilidad.

Para el proceso FIGARCH(p,d,q), a partir de un proceso GARCH(p,q), puede ser expresado como un proceso $\operatorname{ARMA}(\mathrm{m}, \mathrm{p})$ en el cuadrado de los residuos, entonces:

$$
\varepsilon_{t-i}^{2}=\alpha_{0}+\sum_{i=1}^{q} \alpha_{i} \varepsilon_{t-i}^{2}+\sum_{j=1}^{p} \gamma_{j} \varepsilon_{t-j}^{2}-\sum_{j=1}^{p} \gamma_{j} v_{t-j}+v_{t}
$$

entonces

$$
\varepsilon_{t}^{2}=\alpha_{0}+\alpha(L) \varepsilon_{t}^{2}+\beta(L) \varepsilon_{t}^{2}-\beta(L) v_{t}+v_{t}
$$

Luego

$$
(1-\alpha(L)-\beta(L)) \varepsilon_{t}^{2}=\alpha_{0}+(1-\beta(L)) v_{t}
$$

y

$$
v_{t}=\varepsilon_{t}^{2}-h_{t}
$$

donde $m=\operatorname{máx}\{p, q\}$. Por lo cual la dinámica $\operatorname{IGARCH}(\mathrm{p}, \mathrm{q})$ tiene la siguiente estructura:

$$
(1-L) \Phi(L) \varepsilon_{t}^{2}=\alpha_{0}+[1-\beta(L)] v_{t}
$$

donde $\Phi(L)=\sum_{i=1}^{m-1} \Phi_{i} L^{i}$, La cual es de orden $m-1$

En este Modelo $\operatorname{IGARCH}(\mathrm{p}, \mathrm{q})$ dicha persistencia permanente se refiere a la función de autocorrelación de la serie de tiempo, por otro lado en los modelos FIGARCH las caidas de la función de autocorrelación son hiperbólicas, por lo tanto tienden a cero las autocorrelaciones pero lentamente, la persistencia decae lentamente a un ritmo o grado la cual es interpretado por la estimación del parámetro fraccional " $d ", d \in(0,1)$.

El modelo FIGARCH(p,d,m) se define:

$$
(1-L)^{d} \Phi(L) \varepsilon_{t}^{2}=\alpha_{0}+(1-\beta(L)) v_{t}
$$

donde:

$$
\Phi(L)=\sum_{i=1}^{m-1} \Phi_{i} L^{i}
$$

la cual es de orden $m-1$. 
El modelo FIGARCH(p,d,m), los shocks son exactamente la innovación en la representación ARMA del proceso FIGARCH(p,d,m), Asi este shock

$$
\varepsilon_{t}^{2}=\alpha_{0}\left((1-L)^{d} \Phi(L)\right)^{-1}+(1-\beta(L))\left((1-L)^{d} \Phi(L)\right)^{-1} v_{t}
$$

donde $v_{t}$ seria el shock y $(1-\beta(L))\left((1-L)^{d} \Phi(L)\right)^{-1}$ se interpretaría como la persistencia.

Este shock se interpreta como el error de pronótico de la varianza o como la variabilidad de la varianza no explicada.

Si realizamos una analogía de la estructura FIGARCH con la estructura ARFIMA (p,d,q) un modelo lineal homocedástico al igual que en FIGARCH (p,d,q) con alta dependencia entre los datos en donde a diferencia que en el primero la segunda es el operador de la memoria larga sobre la media incondicional $\mu$ de $y_{t}$ del proceso, en el modelo FIGARCH la dependencia es no lineal.

Si a partir de la ecuación (10), $v_{t}=\varepsilon_{t}^{2}-h_{t}$, realizamos un proceso inverso en (13), entonces tendríamos:

$$
(1-L)^{d} \Phi(L) \varepsilon_{t}^{2}=\alpha_{0}+(1-\beta(L))\left(\varepsilon_{t}^{2}-h_{t}\right)
$$

Luego

$$
\begin{aligned}
h_{t} & =\alpha_{0}(1-\beta(L))^{-1}+\left[1-(1-\beta(L))^{-1} \Phi(L)(1-L)^{d}\right] \varepsilon_{t}^{2} \\
& =\alpha_{0}[1-\beta(L)]^{-1}+\sum_{i}^{\infty} \lambda_{i}(L)^{i} \varepsilon_{t}^{2} \\
& =\alpha_{0}[1-\beta(L)]^{-1}+\lambda(L) \varepsilon_{t}^{2}
\end{aligned}
$$

donde $\lambda(L)=\lambda_{1} L+\lambda_{2} L^{2}+\lambda_{3} L^{3}+\cdots$, , con $\lambda_{k} \geqslant 0$ para $k=1,2,3, \ldots \ldots$

Se define $H$ como coeficiente de Hurts (1951), entonces si $2 d-1=2 H-2 \Rightarrow d=H-0,5$, por lo tanto $d$ tiene una relación directa con $H$, es decir a mayor nivel de memoria será mayor valor de $H$, entonces la covarianza de los incrementos tienden a cero en el orden $n^{2 H-2}$ por lo tanto el movimiento Browniano fraccional es un proceso de memoria larga.

$$
\lim _{n \rightarrow \infty} \frac{\rho(n)}{H(2 H-1) n^{2 H-2}}=1
$$

Si $H \in\left(\frac{1}{2}, 1\right)$, entonces $\alpha \in(0,1) \Rightarrow$ MBF (dependencia de largo plazo).

Si $H \in\left(0, \frac{1}{2}\right)$, entonces $\alpha \in(0,1) \Rightarrow$ MBF (no tiene dependencia de largo plazo).

Un proceso estocástico se dice:

1. Persistente, sus trayectoras tienen la misma dirección, es decir, $H \in\left(\frac{1}{2}, 1\right) \Rightarrow \mathrm{d} \in(0,0.5)$ $\Rightarrow H(2 H-1) n^{2 H-2}>0$; los incrementos del MBF tienden a la misma dirección por lo tanto es un proceso persistente. existe dependencia a largo plazo.

2. Antipersistente, sus trayectoras tienden a retroceder a misma, es decir, $H \in\left(0, \frac{1}{2}\right) \Rightarrow \mathrm{d} \in(-0.5,0) \Rightarrow H(2 H-1) n^{2 H-2}<0 ;$ los incrementos del MBF tienden a direcciones opuestas por lo tanto es un proceso antipersistente o memoria corta.

3. $H=\frac{1}{2} \Rightarrow d=0 \Rightarrow H(2 H-1) n^{2 H-2}=0$, los incrementos son independientes hay neutralidad en las direcciones de sus incrementos por lo tanto es un movimiento Browniano. 
Por lo tanto se considera como un proceso no estacionario debido a que un proceso autosimilar es no estacionario pero con incrementos estacionarios e dependientes y esta dependencia es no lineal debido a ser un movimiento browniano fraccionario con $H(2 H-1) n^{2 H-2}>0$ para $H \in(0,5,1)$ existiendo una dependencia a largo plazo o persistencia del shock en la volatilidad (persistencia en la auto correlación de las innovaciones) dichos incrementos tienden la misma dirección las trayectorias tienden a ir en la misma dirección.

$$
d \in \begin{cases}(-0,5,0) & \text { Decimos estacionario antipersistente } \\ (0,0,5) & \text { Decimos estacionario de memoria larga } \\ (0,5,1) & \text { Decimos no estacionario de memoria larga con reversión a la media }\end{cases}
$$

Es importante señalar que si $d \in(-0,5,0,5)$ se garantiza la estacionariedad del proceso. Por lo cual el proceso estocástico con incrementos estacionario y dependientes cuya dependencia es no lineal con índice de similaridad $H \in(0,5,1)$ es un proceso autosimilar con parámetro de memoria larga $d \in(0,0,5)$ con dichos incrementos los cuales tienden a la misma dirección y reversible a la media, pero si $d \in(0,5,1)$ entonces se define como un proceso con incrementos no estacionarios y incrementos dependientes con una menor velocidad en la disminución de la covarianza de los incrementos de los intervalos ajenos, una extensión del movimiento browniano fraccional (memoria larga como la persistencia de la autocorrelación de las innovaciones cuadráticas).

\subsection{La función Impulso respuesta}

La función impulso respuesta se utiliza para analizar y cuantificar el impacto de un shock sobre la volatilidad de una serie de memoria larga, es decir una serie caótica muy sensible a minimos cambios en las condiciones iniciales, teniendo en cuenta que dicho impacto tiende a cero pero en este caso de manera hiperbólica, entonces dicha persistencia de la varianza condicionada es caracterizada en terminos de los coeficientes de dicha función:

$$
\gamma_{k}=\frac{\partial E\left(\varepsilon_{t+k}^{2} \mid \Psi_{t-1}\right)}{\partial \nu_{t}}-\frac{\partial E\left(\varepsilon_{t+k-1}^{2} \mid \Psi_{t-1}\right)}{\partial \nu_{t}}
$$

luego

$$
\gamma_{k}=\frac{\partial h_{t+k}}{\partial \nu_{t}}-\frac{\partial h_{t+k-1}}{\partial \nu_{t}}
$$

A partir de $v_{t}=\varepsilon_{t}^{2}-h_{t}$ resulta

$$
\gamma_{k}=\frac{\partial h_{t+k}}{\partial \nu_{t}}-\frac{\partial h_{t+k-1}}{\partial \nu_{t}}=L(\lambda)\left\{\frac{\partial E\left(\varepsilon_{t+k}^{2} \mid \Psi_{t-1}\right)}{\partial \nu_{t}}-\frac{\partial E\left(\varepsilon_{t+k-1}^{2} \mid \Psi_{t-1}\right)}{\partial \nu_{t}}\right\} .
$$

Entonces para encontrar los coeficientes de la función impulso respuesta se tendrá lo siguiente

$$
\varepsilon_{t}^{2}-v_{t}=\alpha_{0}(1-\beta(L))^{-1}+\left[1-(1-\beta(L))^{-1} \Phi(L)(1-L)^{d}\right] \varepsilon_{t}^{2}
$$

luego

$$
\begin{aligned}
\varepsilon_{t}^{2} & \left.\left.=-\left[\alpha_{0}(1-\beta(L))^{-1}\right]\left[(1-\beta(L)) \Phi(L)^{-1}(1-L)^{-d}\right)\right]-v_{t}\left[(1-\beta(L)) \Phi(L)^{-1}(1-L)^{-d}\right)\right] \\
& \left.\left.=-\left[\alpha_{0}(\Phi(L))^{-1}\right]\left[\Phi(L)(\Phi(L))^{-1}(1-L)^{-d}\right)\right]-v_{t}\left[\beta_{k}(\Phi(L))^{-1}(1-L)^{-d}\right)\right] \\
& \left.\left.=-\left[\alpha_{0}(\Phi(L))^{-1}\right]\left[(1-L)^{-d}\right)\right]-v_{t}\left[\beta_{k}(\Phi(L))^{-1}(1-L)^{-d}\right)\right]
\end{aligned}
$$


y

$$
\begin{aligned}
\frac{\partial E\left(\varepsilon_{t+k}^{2} \mid \Psi_{t-1}\right)}{\partial \nu_{t}} & \left.=-\left[\beta_{k}(\Phi(L))^{-1}(1-L)^{-d}\right)\right] L(\lambda) \\
& \left.=-\left[\beta_{k}(\Phi(L))^{-1}(1-L)^{-d}\right)\right]\left[1-(1-\beta(L))^{-1} \Phi(L)(1-L)^{d}\right] \\
& \left.=-\left[\beta_{k}\left[1-(1-\beta(L))^{-1} \Phi(L)(1-L)^{d}\right](\Phi(L))^{-1}(1-L)^{-d}\right)\right] \\
& \left.=-\beta_{k}(\Phi(L))^{-1}(1-L)^{-d}\right)+\beta_{k}(1-L)^{-d} \\
& \left.==-\beta_{k-1}(\Phi(L))^{-1}(1-L)^{-d}\right)+\beta_{k}(1-L)^{-d} .
\end{aligned}
$$

Por lo tanto,

$$
\left.\left.\gamma_{k}=-\beta_{k}(\Phi(L))^{-1}(1-L)^{-d}\right)+\beta_{k-1}(\Phi(L))^{-1}(1-L)^{-d}\right)-\beta_{k}(1-L)^{-d}
$$

luego

$$
\gamma_{k}=\left(\beta_{k-1}-\beta_{k}\right)(\Phi(L))^{-1}(1-L)^{-d}
$$

Lo que demuestra que en el modelo Figarch $(1, \mathrm{~d}, 1)$, los coeficientes de la función impulso respuesta no dependen del tiempo $t$, los cuales indican la persistencia de la varianza condicionada (Baillie, 1996).

El término ruido blanco fraccionario tambien puede expresarse como una función hipergeométrica de Gauss:

$$
(1-L)^{d}=F(-d, 1,1 ; L)
$$

$\mathrm{y}$

$$
F(-d, 1,1 ; L)=\sum_{k=0}^{\infty} \frac{(-d)_{k}(1)_{k}}{k !(1)_{k}} L^{k}=\sum_{k=0}^{\infty} \frac{(-d)_{k}}{k !} L^{k}
$$

El termino $(a)_{k}$ se denomina símbolo de Pochhammer, donde:

$$
(a)_{k}= \begin{cases}1 & \text { si } k=0 \\ a(a+1) \ldots(a+k-1) & \text { si } k=1,2,3, \ldots\end{cases}
$$

Además

$$
\sum_{k=0}^{\infty} \frac{\Gamma(-d+k)}{\Gamma(-d) k !} L^{k}=\sum_{k=0}^{\infty} \frac{\Gamma(k-d)}{\Gamma(k+1) \Gamma(-d)} L^{k}
$$

Por lo tanto, si combinamos las ecuaciones (17) y (18), es decir tomando en cuenta la persitencia del shock, resulta

$$
\begin{aligned}
\gamma_{k} & =(1-\beta(L))(\Phi(L))^{-1}(1-L)^{-d} \\
& =(1-\beta(L))(\Phi(L))^{-1} F(-d, 1,1 ; L)
\end{aligned}
$$

Por lo tanto, la función impulso respuesta acumulativa se expresa:

$$
\gamma_{1}=\varphi(0)+\varphi(1)+\varphi(2)+\cdots=\lim _{s \rightarrow \infty} \sum_{i=0}^{s} \varphi(i)
$$

Si definimos la ecuación 19 como $F(d-1,1,1, L)=(1-L)^{1-d}$, para $0 \leqslant d<1$, entonces se presentan los siguientes casos: 
Caso $d=0$ se genera un $\operatorname{GARCH}(\mathrm{p}, \mathrm{q})$ memoria corta relacionado con el proceso gaussiano con incrementos estacionario e independientes con índice de similaridad $H=\frac{1}{2}$ (movimiento browniano), la función impulso respuesta acumulativa es:

$$
\begin{aligned}
& \gamma_{1}=\left(1-\beta_{1}(1)\right)\left(\Phi_{1}(1)\right)^{-1} F(d-1,1,1 ; 1) \\
& \gamma_{1}=0 \\
& F(d-1,1,1 ; 1)=0 \\
& \qquad \lim _{s \rightarrow \infty} \sum_{i=0}^{s} \varphi(i)=0
\end{aligned}
$$

La varianza condicionada se estabiliza automáticamente, la covarianza de intervalos ajenos desaparece exponencialmente.

Caso $d=1$ se genera un $\operatorname{IGARCH}(\mathrm{p}, \mathrm{q})$ memoria persistente y d raices unitarias,La función impulso respuesta acumulativa es:

$$
\begin{aligned}
& \gamma_{1}=\left(1-\beta_{1}(1)\right)\left(\Phi_{1}(1)\right)^{-1} F(d-1,1,1 ; 1) \\
& \gamma_{1}=\left(1-\beta_{1}(1)\right)\left(\Phi_{1}(1)\right)^{-1} \\
& F(d-1,1,1 ; 1)=1
\end{aligned}
$$

$$
\lim _{s \rightarrow \infty} \sum_{i=0}^{s} \varphi(i)=\left(1-\beta_{1}(1)\right)\left(\Phi_{1}(1)\right)^{-1} .
$$

La persistencia es indefinida, el efecto de un shock persiste indefinidamente.

Caso $0<d<1$ se genera un FIGARCH(m,d,q) memoria larga relacionado con el proceso con incrementos estacionarios y dependientes o no estacionarios y dependientes tales situaciones determinados por $d$ con índice de similaridad $H>\frac{1}{2}$, La función impulso respuesta acumulativa es:

$$
\begin{aligned}
& \gamma_{1}=0 \\
& F(d-1,1,1 ; 1)=0
\end{aligned}
$$

$$
\lim _{s \rightarrow \infty} \sum_{i=0}^{s} \varphi(i)=0 .
$$

La persistencia desaparece hiperbólicamente tiende a cero al igual que garch pero la diferencia es la velocidad de las disminuciones de las autocorrelaciones y la serie revierte a su media.

Caso $d>1$, La función impulso respuesta acumulativa es:

$$
\begin{aligned}
& \gamma_{1}=\left(1-\beta_{1}(1)\right)\left(\Phi_{1}(1)\right)^{-1} F(d-1,1,1 ; 1) \\
& \gamma_{1}=\infty \\
& F(d-1,1,1 ; 1)=\infty \\
& \qquad \lim _{s \rightarrow \infty} \sum_{i=0}^{s} \varphi(i)=\infty
\end{aligned}
$$

La varianza condicionada es explosiva e irreal.

\section{Datos y Variables}

Para la aplicación del cuerpo teórico desarrollado sirvieron como insumos numéricos la serie de tiempo tipo de cambio diario nominal dólar-sol de un periodo inicial del año 1997 al 2017, para el cual se consideró desde el mes de enero de 1997 al mes de marzo del 2017, dicha muestra sirvió para la estimación y validación del modelo. 
La estimación del modelo FIGARCH para la data existente es la siguiente:

$$
\begin{gathered}
r_{t}=100 \log \left(s_{t} / s_{t-1}\right)=\mu+\epsilon_{t} \\
\epsilon_{t} h_{t}^{-1 / 2} \sim N(0,1) \\
h_{t}=\alpha_{0}+\beta_{1} h_{t-1}+\left[1-\beta_{1} L-\left(1-\phi_{1} L\right)(1-L)^{d}\right] \epsilon_{t}^{2}, t=1,2, \ldots, 5265
\end{gathered}
$$

\section{Resultados Metodológicos}

En esta sección realizamos la estimación del modelo de memoria larga FIGARCH es decir un proceso estocástico gaussiano de tipo movimiento browniano fraccional con parámetro de memoria larga "d. ${ }^{a}$ partir de una serie de tiempo obtenido del Banco central de reserva del Perú de tipo de cambio diario nominal dólar-sol desde el 3 de Enero de 1997 hasta el 9 de Marzo del 2017, generando un total de 5625 observaciones.

\subsection{Prueba de Coeficiente de Hurts}

En el tabla 1 se muestra el coeficiente de hurts de la serie del cuadrado de los retornos el cual me indica un valor de 0.76409 siendo mayor a 0.5 y menor a 1 entonces indicaria la sospecha de un movimiento browniano fraccionario con un parámetro de memoria larga $d>0$ por lo tanto la dependencia a largas distancias de tiempo en los retornos porcentuales al cuadrado de dicha serie, es decir memoria larga en la dinámica de la varianza condicionada, se consideraría dicha serie como caótica muy sensible a cambios en las condiciones iniciales, es decir, la tendencia de la serie seria la misma al periodo anterior. Se tendrá un 76.5 por ciento de probabilidad de que la tendencia sea la misma del periodo anterior.

Tabla 1. Coeficiente de Hurts

\begin{tabular}{|c|c|}
\hline Serie & Exponente de Hurst estimado \\
\hline cuadrado de los retornos & 0.764095 \\
\hline retornos porcentuales & 0.654963 \\
\hline
\end{tabular}

Como referencia, en la figura 1 se representa los retornos porcentuales del tipo de cambio bajo consideración, la cual indica la existencia de la volatilidad de la serie en el periodo de análisis, del mismo modo la función de auto correlación del cuadrado de los retornos indica una caída hiperbólica como se muestra en la figura 2, indicando la posibilidad de modelar la serie con un modelo de memoria larga cuyas pruebas no paramétricas y semiparamétricas confirman.

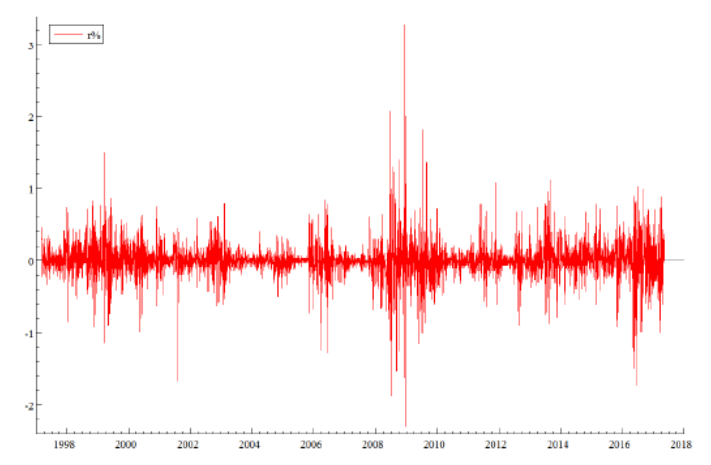

Figura 1. Retorno porcentuales del tipo de cambio 


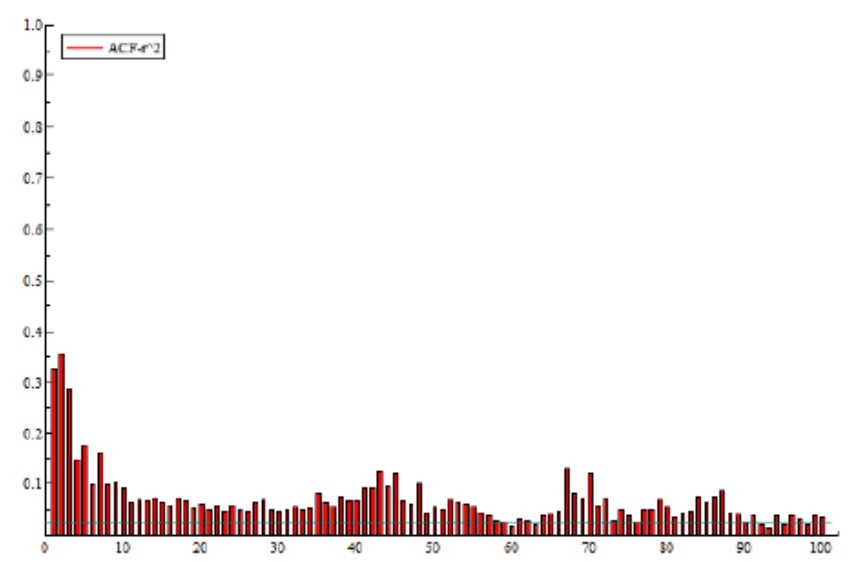

Figura 2. Función de autocorrelación del cuadrado de los retornos

Tabla 2. Estimación de modelos FIGARCH

\begin{tabular}{|c|c|c|c|}
\hline$(\mathrm{p}, \mathrm{d}, \mathrm{q})$ & $(0,0,0)$ & $(1, \mathrm{~d}, 0)$ & $(1, \mathrm{~d}, 1)$ \\
\hline$\mu$ & 0.001678 & -0.008167 & -0.007849 \\
& $(0.0036107)$ & $(0.0018807)$ & $(0.0018779)$ \\
$\alpha_{0}$ & 0.058414 & 0.000133 & 0.000165 \\
$\beta_{1}$ & - & 0.262035 & 0.647364 \\
& & & $(0.075995)$ \\
$\phi_{1}$ & - & - & 0.293989 \\
& & & $(0.073052)$ \\
$\mathrm{d}$ & - & 0.527594 & 0.649139 \\
& & $(0.05746)$ & $(0.07466)$ \\
& & & \\
$\mathrm{S}$ & 0.65719 & 0.18541 & 0.23524 \\
$\mathrm{~K}$ & 18.943 & 5.3988 & 5.5193 \\
$\mathrm{Q}(20)$ & 148.662 & 145.449 & 135.622 \\
$Q(20)^{2}$ & 2069.97 & 17.6941 & 14.4002 \\
LogLikelihood: & 5.005 & 1697.577 & 1714.682 \\
\hline
\end{tabular}

La estimación del modelo FIGARCH para la data existente es la siguiente:

$$
h_{t}=0,000165+0,647364 h_{t-1}+\left[1-0,647364 L-(1-0,293989 L)(1-L)^{0,649139}\right] \epsilon_{t}^{2}
$$

En este caso se aplicar el cuerpo teorío del movimiento browniano fraccional mediante la modelación de los retornos porcentuales logarítmicos $r_{t}$ los cuales tienen incrementos dependientes a largo plazo. La estimación es mediante la QMLE bajo la suposición de la normalidad condicional, en el tabla 2 entre paréntesis se indican los errores estandar robustos de cada estimación en dicho cuadro se indican los modelos estimados de figarch, los cuantiles $Q(20)$ y $Q(20)^{2}$ se 
refieren a la prueba de Ljung Box Portmanteau de la correlación serial de hasta el orden 20 en los residuos estandarizados y los residuos estandarizados al cuadrado respectivamente, en cada columna se describen las estimaciones de cada modelo, en la primera columna se describe al modelo $(p, d, q)=(0,0,0)$ la cual corresponde al modelo $\operatorname{GARCH}(0,0)$, en la segunda y tercera columna se especifican diferentes modelos FIGARCH. En las cuales se puede notar que el valor de logaritmo de verosimilitud de $\operatorname{FIGARCH}(1, \mathrm{~d}, 1)$ es más alto dicho valor me indica entonces la idoneidad del modelo FIGARCH $(1, \mathrm{~d}, 1)$.

Por otro lado si usamos los criterios de información dados en la tabla 3 , el modelo FIGARCH $(1,0.65,1)$, es el proceso que mejor se adapta a la data en análisis de tipo de cambio debido al menor valor comparando entre todos los criterios de información de cada uno de los modelos estimados indicados en la tabla 3, por lo tanto se fortalece la propuesta del modelo FIGARCH $(1,0.65,1)$ como el modelo más idóneo para el conjunto de datos existente.

Si analizamos la tabla 4, se indican dos de las diferentes medidas que se utilizan para comparar la precisión entre modelos de pronóstico MSE (error cuadrático medio) y RSME(raíz del error cuadrático medio) que corresponde al pronóstico de la varianza condicional. Se nota que el modelo FIGARCH $(1,0.65,1)$ da los mejores pronósticos representados en mínimos valores en MSE Y RMSE con valores de 0.00015110 .01229 respectivamente.

Tabla 3: Criterio de información para la elección del modelo

\begin{tabular}{|c|c|c|c|c|}
\hline$(\mathrm{p}, \mathrm{d}, \mathrm{q})$ & Akaike & Schwarz & Shibata & Hannan-Quinn \\
\hline$(0,0,0)$ & -0.001341 & 0.001519 & -0.001342 & -0.000333 \\
$(1,0.53,0)$ & -0.755892 & -0.750173 & -0.755894 & -0.753877 \\
$(1,0.65,1)$ & -0.763081 & -0.755931 & -0.763083 & -0.760561 \\
\hline
\end{tabular}

Si consideramos un medida de selección entre modelos de pronostico adicional se tiene en la tabla 4, el coeficiente de desigualdad de Theil la cual me permite analizar la efectividad del modelo de predicción seleccionado cuyo valor puede ser mayor a 1 , cuanto más cercano a cero mejor es la efectividad del modelo, es decir entre los tres modelos estimados se elegirá el más cercano a cero, en la tabla 4 los modelos $\operatorname{FIGARCH}(1,0.53,0)$ con un valor de 0.345 y el modelo FIGARCH $(1,0.65,1)$ con valor de 0.346 son casi de la misma efectividad, pero se podría concluir en función a los análisis anteriormente realizados que a pesar de ser marginalmente mayor coeficiente de Theil pero en vista de la evidencia de las anteriores medidas me indicarían, que el mejor modelo seria el proceso browniano fraccional de $\operatorname{FIGARCH}(1,0.65,1)$ o de memoria larga.

Tabla 4. Medidas de error de los pronósticos de la varianza condicional

\begin{tabular}{|c|c|c|c|}
\hline$(\mathrm{p}, \mathrm{d}, \mathrm{q})$ & MSE & RMSE & TIC \\
\hline$(0,0,0)$ & 0.002162 & 0.0465 & 0.614 \\
$(1,0.53,0)$ & 0.0001604 & 0.01266 & 0.3449 \\
$(1,0.65,1)$ & 0.0001511 & 0.01229 & 0.3457 \\
\hline
\end{tabular}

En la siguiente tabla 5, se muestra la prueba de Engle (1982) de los residuales estandarizados al cuadrado en la cual se acepta la hipótesis nula de homocedasticidad en los residuos debido a que la probabilidad asociada al estadístico nR2 es mayor a 0.05 entonces confirma la no existencia de posible correlacion serial significativa en los residuales.

En concordancia con Hsieh(1989), dicha varianza condicionada está bien especificada cuando 
Tabla 5. ARCH test on Squared Standardized

Residuals

\begin{tabular}{|c|c|c|c|}
\hline ARCH test: & $\mathrm{F}(2,4474)$ & 0.10625 & {$[0.8992]$} \\
\hline ARCH test: & $\mathrm{F}(5,4468)$ & 1.7345 & {$[0.1231]$} \\
\hline ARCH test: & $\mathrm{F}(10,4458)$ & 1.052 & {$[0.3964]$} \\
\hline
\end{tabular}

Tabla 6. Q-Statistics on Squared Standardized Residuals Ljung-Box

\begin{tabular}{|l|c|c|}
\hline $\mathrm{Q}(10)=$ & 10.9355 & {$[0.2053786]$} \\
\hline $\mathrm{Q}(20)=$ & 14.4002 & {$[0.7026523]$} \\
\hline $\mathrm{Q}(50)=$ & 35.917 & {$[0.9006973]$} \\
\hline
\end{tabular}

la excesiva curtosis de los residuales estandarizados no exceden a la excesiva de curtosis de los residuales no estandarizados. Entonces para dicho fenómeno en estudio, la excesiva curtosis de los residuales estandarizados con 4.5 no excede a la excesiva de curtosis de los residuales no estandarizados con 18.9, por lo tanto el modelo está muy bien especificado.

En la siguiente tabla 6, se muestra la ausencia de autocorrelación de la serie transformada mediante la prueba de Ljung- Box, por lo tanto se dan señales de que dicho modelo construido para la dinámica de la volatilidad captura adecuadamente la estructura de correlación debido a que la probabilidad asociada es mayor al 0,05 aceptandose la hipótesis nula de ausencia de autocorrelación.

Por lo tanto la varianza condicionada estimada está representada en la figura 3.
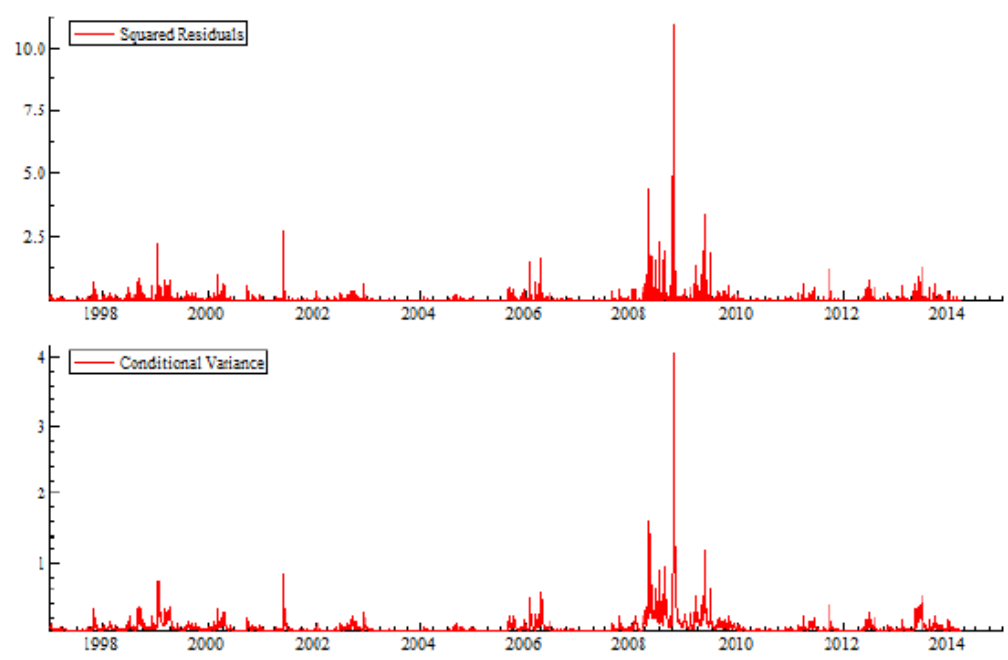

Figura 3. La varianza condicional estimada 


\section{Conclusiones}

1. Se comprueba la persistencia de los shocks que tiene una durabilidad a largo plazo al evidenciar empíricamente un orden de integración fraccional de $d=0,6491$ en su volatilidad.

2. Como consecuencia de la estimación del parámetro de memoria larga $d>0$ se identifica al proceso de varianza condicional como un proceso autosimilar con $H>0$ con un coeficiente de Hurts $H=0,764095$ en los retornos cuadráticos $H \neq \frac{1}{2}$ evidenciando una mayor persistencia en la volatilidad de esta manera se concluye rechazando la naturaleza del proceso como un movimiento browniano identificando de este modo al proceso como un movimiento browniano fraccional no estacionario con incrementos no estacionarios debido a que el parámetro de memoria larga $d=0,6491$ se encuentra fuera del rango $(-0,5,0,5)$ dicho rango garantiza la estacionariedad de sus incrementos y con incrementos dependientes cuya dependencia es no lineal. De esta manera se logra el objetivo de identificar al proceso de varianza condicional como un movimiento browniano fraccional (sistema caótico).

3. Se logró la estimación y la especificación del modelo Autorregresivo con Heterocedasticidad condicionada Generalizada Fraccionalmente integrado del tipo de cambio nominal del Perú - FIGARCH(1,d,1) señalado en la tabla 2.

$$
h_{t}=0,000165+0,647364 h_{t-1}+\left[1-0,647364 L-(1-0,293989 L)(1-L)^{0,649139}\right] \epsilon_{t}^{2}
$$

para $t=1,2, \cdots, 5265$

El modelo FIGARCH $(1,0.65,1)$ cumple con todos los test de significancia de sus parámetros y las desigualdades dadas por Baillie(2001) para garantizar la no negatividad de la varianza condicionada. Según el criterio de Hsieh(1989) dicha varianza condionada esta bien especificada debido a que la excesiva curtosis de los residuos estandarizados con un valor de 4.5 no excede al exceso de curtosis de los residuos no estandarizados con un valor de 18.9 .

4. En relación a los modelos de volatilidad estimados se muestran buenos resultados en el modelo FIGARCH $(1,0.53,1)$ y aceptables en el modelo $\operatorname{GARCH}(0,0)$, sin embargo debido a que en el modelo FIGARCH $(1,0.53,1)$ el valor de logaritmo de verosimilitud es menor que en modelo FIGARCH $(1,0.65,1)$ y en el modelo $\operatorname{GARCH}(0,0)$ el valor de logaritmo de verosimilitud es significativamente de menor que en modelo $\operatorname{FIGARCH}(1,0.65,1)$ se demuestra la idoniedad del modelo propuesto $\operatorname{FIGARCH}(1,0.65,1)$ como se indica en la tabla 2 los cuales son confirmados por los criterios de información de la tabla 3.

5. Mediante la función impulso respuesta acumulativa se cuantificó y demostró la durabilidad del shock en la volatilidad de la varianza condicionada que tiende a cero pero hiperbólicamente a una tasa de $n^{0,649139}$.Se utilizó la función hipergeométrica para explicar el ruido blanco fraccionario $F(d-1,1,1 ; 1)=0$ y $\operatorname{lím}_{s \rightarrow \infty} \sum_{i=0}^{s} \varphi(i)=0$.

\section{Sugerencias para futuras investigaciones}

1. Es importante mencionar que existen investigaciones como la de Sebastián Laurent y Michael Baine(2000) los cuales indican que la posible existencia de memoria larga en la serie es debido a un posible quiebre estructural, encontrando evidencia que sustenta la hipótesis de que la existencia de un posible quiebre estructural generaría un incremento en la memoria de la serie, debido a la extensión que demandaría incluir dicho análisis solo mencionó este fenómeno para futuras investigaciones. 
2. Con respecto a la relacion quiebre estructural y memoria larga Baillie y Morata(2009) desarrollan un nuevo proceso de memoria larga que incluye la memoria a largo plazo y quiebre estructural en el mecanismo de volatilidad denominado A-FIGARCH(p,d,q,k) (Adaptive FIGARCH) dicho modelo constituye un componente estocásticos de memoria larga y otro componente determinístico de proceso de quiebre, dicho tema es sujeto en la actualidad de investigaciones en Nash Boutahr y Travbelsi(2010) se presenta mucha mas información de estructuras de A-FIGARCH Y derivados como FITVGARCH.

\section{Referencias bibliográficas}

[1] Billingsley, P.(1968). Convergence of Probability Measures. New York, United States: John Wiley and Sons .

[2] Briones, J. (2018). Modelo autorregresivo con heterocedasticidad condicionada generalizada fraccionalmente integrado (tesis de maestría). Universidad Nacional Mayor de San Marcos, Perú.

[3] Brockwell P. J. and Davis R.A.Springer Verlag (1987). Time Series Theory and Methods. Paris, Francia: Mason.

[4] C. Fox. (1928). The asymptotic expansion of generalized hypergeometric functions. Proceedings of the London Mathematical Society 2(27), 389 - 400.

[5] Engle, R. (1982) Autoregressive conditional heterocedasticity with estimates of the variance of United Kingdom inflations. Econometrica, 50(4), 987-1007.

[6] Feller, W. (1971). An Introduction to Probability Theory and its Applications .New York,United States: John Willey and Sons,

[7] Granger, C. W. J. (1980). Long memory relationships and the aggregation of dynamic models. Journal of Econometrics, 14(2) pp. 227-238.

[8] L. Decreusefond and A.S. üstünel (1999). Stochastic analysis of fractional Brownian motion. Potential analysis, 10(2), 177-214. 